\title{
Vergleichende Analyse der Phonologie der nördlichen Mundarten der obugrischen Sprachen
}

0. Im folgenden werden einige Fragen der Lautgeschichte der nördlichen wogulischen und ostjakischen Mundarten behandelt. Ich sehe dies als eine verlockende Arbeit an, da die Systeme der Vokalphoneme in den benachbarten nördlichen Mundarten der beiden Sprachen völlig übereinstimmen, welcher Umstand gewisse fehlerhafte Schlussfolgerungen verursacht hat.

Diese Übereinstimmung ist in der Finnougristik allgemein bekannt. Wolfgang Steinitz war der Meinung, dies könne die Folge der geographischen Nachbarschaft sein. Steinitz hat eigentlich öfters auf diese Deutungsmöglichkeit hingewiesen; er hat aber nie versucht, diese Vermutung zu beweisen. So möchte ich jetzt näher untersuchen, ob es sich wirklich um eine Übereinstimmung handelt, die von zum Teil gemeinsamen nordwogulischen und nordostjakischen Lautveränderungen herrührt oder ob sie als zufälliges Produkt voneinander unabhängiger Lautentwicklungen in beiden Sprachen betrachtet werden soll.

0.1. Obgleich es sehr interessant ist, die sekundär gemeinsamen Züge in miteinander verwandten Sprachen oder deren Mundarten zu erforschen, ist diese Untersuchung jedoch kein Selbstzweck. Ihre besondere Aktualität ist János Gulya zu verdanken, der u.a. aufgrund der gemeinsamen Vokalphonemsysteme in den nördlichen obugrischen Mundarten den Schluss gezogen hat, nach dem "'a kutatások jelen állásában a kettéosztódásos magyarázat helyett célszerübb közvetlenül három későbbi ugor jellegü nyelv proto-nyelvi formáját, azaz ősmagyart, 
ősvogult és ősosztjákot, illetőleg eleve magyart, vogult és osztjákot minden további összekapcsolás nélkül felvennünk" (Gulya 1977. 118). Bei der Betrachtung der linguistischen historischen Fragen, die mit der ugrischen Grundsprache verknüpft sind, behauptet er (ebd.) unter Berufung auf Steinitz (STEINItz 1955. 152), die Ähnlichkeiten im Bereich der Lautlehre seien auf gegenseitige Einflüsse zurückzuführen und nach ihm: 'Ilyen egyezések a hangtanon kívül e nyelvekben másutt is vannak (pl. a birtokos személyjelek körében)" (GULYA 1977. 118).

An der von Gulya angegebenen Stelle schreibt Steinitz: ,'Eine wichtige Hilfe für die Feststellung des urwog. Zustandes und der wog. Vokalgeschichte, insbesondere eine unentbehrliche Hilfe für die vorwog. Vokalgeschichte kann schliesslich der Vergleich mit dem ostjakischen Vokalismus geben, dessen Geschichte verhältnismässig gründlich erforscht und geklärt ist und der gegenüber dem wog. Vokalismus im Allgemeinen einen archaischeren Zustand vertritt' (STEINITZ 1955. 152). Wer das zitierte Buch von Steinitz ein wenig studiert hat, muss unbedingt darüber im klaren sein, dass es sich hier um die Entscheidung für entweder urwog. ${ }^{*} \bar{e}$ oder urwog. ${ }^{*} \breve{e}$ handelt (vgl. Steinitz 1955. 205, 265, 266). An der zitierten Stelle finden wir also keinerlei Hinweis auf den ''gegenseitigen sekundären Einfluss"' (so Gulya 1977. 118), so unterstützt Steinitz hier keineswegs die von Guyla aufgestellten Thesen, nach denen es keine obugrische Grundsprache gegeben hätte und die engeren Beziehungen zwischen dem Wogulischen und dem Ostjakischen ausschliesslich der Nachbarschaft und den späteren Berührungen zwischen ihnen zu verdanken wären.

1. Gulya hat vielleicht an die tatsächlich existierenden sekundär gemeinsamen Züge einiger Mundarten der beiden Sprachen gedacht.

In seiner Geschichte des wogulischen Vokalismus brachte Steinitz die ähnlichen Züge der Phonemsysteme des Wogulischen an der unteren Konda und des Ostjakischen an der Konda in Zusammenhang (Steinitz 1955. 164). Da aber einerseits das von Steinitz aufgestellte System der Konda-wogulischen Vokalphoneme revidiert werden muss, andererseits es auch tiefge- 
hende Unterschiede zwischen ihnen gibt, die bei einem Vergleich nicht ausser acht gelassen werden dürften, beschränke ich mich auf das Nordwogulische und auf das Nordostjakische (meine Auffassung über die Vokalphoneme der KU Mundart s. HonTi 1979).
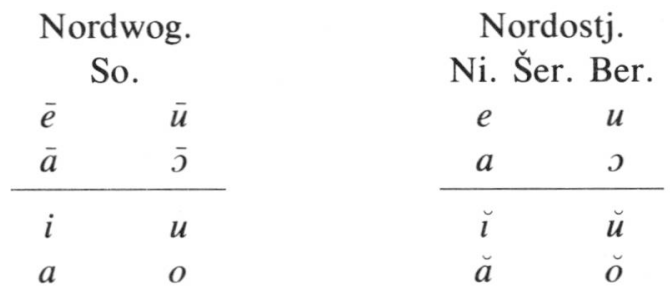

(S. Steinitz 1955. 166, 167.) Das System in der ostj. Kaz. Mundart, die unmittelbare Kontakte zum Nordwogulischen hat, unterscheidet sich von diesem (gemein-)nordostjakischen System dadurch, dass statt $u$ in ihr ein $o$, "ein eigenartiger, geschlossener, etwas palatalisierter $o$-Laut' (STEINITZ 1950. 16) $\mathrm{zu}$ finden ist.

Steinitz bemerkte diese Übereinstimmung bereits vor langem und versuchte sie mit der Ostjakisierung von Wogulen bzw. mit ostjakischem Substrat im Wogulischen zu erklären (STEINITZ 1937. 277, hierzu vgl. noch Steinitz 1941/1976. 64). Ungefähr zwanzig Jahre später vertrat er immer noch dieselbe Meinung: "Die Entstehung des Vokalsystems im Nordwog. lässt sich zwar aus inneren Tendenzen der wogulischen Sprachentwicklung erklären. Sie kann aber zweifellos nur im Zusammenhang mit dem völlig übereinstimmenden Vokalsystem der benachbarten ostjakischen Dialekte von Scher., Ber. und Ni. und seiner teilweise bis in die Einzelheiten übereinstimmenden Geschichte verstanden werden" (STEINITZ 1955. 167).

Es wäre nützlich zu wissen, bis wann sich die obigen Systeme herausbildeten, seit wann und in welchem Masse sich die gegenseitigen Beziehungen des Nordwogulischen und Nordostjakischen entfalteten. Wenn wir diese Fragen geklärt hätten, wäre es möglich, beruhigend zu klären, ob es sich tatsächlich um Wechselwirkungen handelt oder die gemeinsamen Züge 
sozusagen zufälligerweise zustandegekommen sind. Die diesbezüglichen historischen Kenntnisse sind spärlich; so sind wir gezwungen zu versuchen, diese Frage ausschliesslich mit Hilfe der Lautgeschichte zu beantworten.

Vor allem möchte ich klären, ob sich die auffallende Übereinstimmung der Vokalphonemsysteme der ersten Silbe im Nordwogulischen und im Nordostjakischen durch innersprachliche Änderungen ableiten lässt (welche Möglichkeit auch von Steinitz nicht ausgeschlossen worden ist, s. oben).

1.1. Das urostjakische Vokalsystem ist dem in $\mathrm{VVj}$. gleich (STEINITZ 1950 a. 48):

\begin{tabular}{cccc}
$i$ & $u$ & $i$ & $\ddot{u}$ \\
& $o$ & $e$ & $\ddot{o}$ \\
$a$ & $\jmath$ & $\ddot{a}$ & $\ddot{\jmath}$ \\
\hline$\breve{a}$ & $\breve{o}$ & $\breve{e}$ & $\breve{o}$
\end{tabular}

Daraus hat sich das urwestostjakische System durch die Etappen entwickelt:

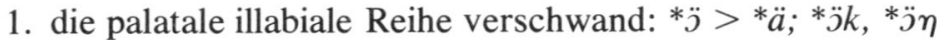

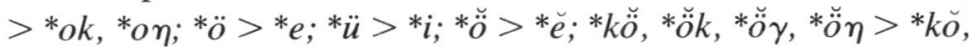
$* \breve{o k},{ }^{*} \breve{o} \gamma, * o \eta$

2. die vollen (langen) velaren weiten und mittelweiten fielen zusammen: ${ }^{*} \jmath \times *^{*}>{ }^{*} o$;

3. $*_{i}>i$.

Das urwestostjakische System (vgl. STEInitz 1950 a. 51-53):

\begin{tabular}{cc}
$u$ & $i$ \\
$o$ & $e$ \\
$a$ & $\ddot{a}$ \\
\hline$\check{o}$ & $\breve{e}$ \\
$\breve{a}$ &
\end{tabular}

Dies stimmt im wesentlichen mit dem in der Mundart von Obdorsk $(\mathrm{O})$ überein; die später eingetretenen Lautveränderungen haben ja nur die Belastung der Phoneme modifiziert (vgl. Steinitz 1950 a. 52). Das System der südlichen Mundarten hat sich durch die positionsbedingten Lautwandel ${ }^{*} a>a, o,{ }^{*} o>o$, $u,{ }^{*} u>u, \ddot{u}, * \breve{e}>\breve{e}, \stackrel{a}{a}$ herausgebildet (vgl. Steinitz 1950 a. 53). 
Im Nordostjakischen kam ein fast vollkommen harmonisches System durch kombinatorische Lautveränderungen und Phonemspaltungen zustande:

\begin{tabular}{cc}
$u$ & $i$ \\
$o$ & $e$ \\
$a$ & $\ddot{a}$ \\
\hline$\breve{u}$ & $\check{\imath}$ \\
$\check{o}$ & \\
$\breve{a}$ & $\breve{a}$
\end{tabular}

Von dieser Etappe an führen drei weitere Entwicklungstendenzen bis zum gegenwärtigen Nordostjakischen:

1. die vollen Engvokale wurden reduziert (kurz): ${ }^{*} i>\breve{i}$ (aber $*_{i \gamma}>$ Ni. Šer. $\left.e \chi\right),{ }^{*} u>\breve{u}\left(\right.$ aber ${ }^{*} u \gamma>$ Ni. Šer. $\left.o \chi\right)$; diese Wandlung ist in O immer noch im Gange (vgl. Steinitz 1950 a. $52-53)$;

2. die velaren weiten Vokale sind labialisiert worden: ${ }^{*} a>0$, $* \breve{a}>\breve{o}$ (aber $* \breve{a} \chi, * \breve{a} \eta \chi>\breve{a} \chi, \breve{a} \eta \chi)$;

3. die palatalen weiten Vokale sind zu hinten gebildeten worden: $* \ddot{a}>a, * \breve{a}>\check{a}$.

Allein das ${ }^{*} o$ bedarf einer Erklärung, an dessen Stelle $o$ in Kaz., $u$ in den übrigen Mundarten zu finden ist: im System mit zwei Öffnungsgraden ist der mit $o$ bezeichnete Laut der Engvokal, der eigentlich wie ein weiter $\bar{u}$-Vokal $(u)$ klingt (Steinitz 1950 b. 37; Rédei 1961. 4; Honti 1978. 140). Also:
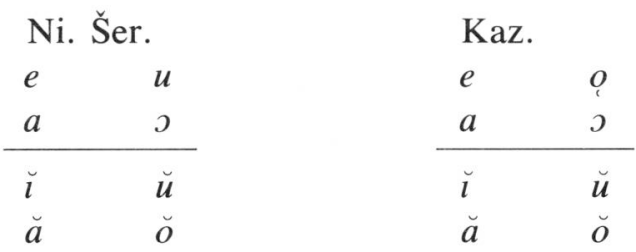

1.2. Und nun betrachten wir die Herausbildung des nordwogulischen Vokalismus!

Von Steinitz' Auffassung (StEINITZ 1955. 154) abweichend 
habe ich das urwogulische Vokalsystem wie folgt erschlossen (Honti i. D.):

\begin{tabular}{cccc}
$\bar{l}$ & $\bar{u}$ & $\bar{l}$ & $\bar{u}$ \\
$\bar{a}$ & & $\overline{\ddot{a}}$ & \\
\hline$\breve{b}$ & $\breve{u}$ & $\breve{l}$ & $\breve{u}$ \\
$\breve{a}$ & $\breve{\jmath}$ & $\breve{a}$ &
\end{tabular}

Die Unterschiede bestehen darin, dass bei mir die mittelweiten $*_{\bar{e}}$ und $*_{\breve{e}}$ fehlen; statt ihrer habe ich aufgrund der interdialektalen Entsprechungen und innerdialektalen Verhältnisse für das Urwogulische $* \breve{a}$ angesetzt; dem $* \check{a}$ von Steinitz entspricht bei mir $* \bar{a}$. Ausserdem habe ich die Bezeichnung einiger urwogulischer Vokale etwas modifiziert: ${ }^{*} \bar{e} \rightarrow{ }_{\bar{l}}^{*},{ }^{*} \breve{o} \rightarrow$ *ว.

Das nordwogulische System ist durch die folgenden Änderungen, die sich zum Teil auch auf andere Dialekte erstreckten, aus dem obigen entstanden:

1. die palatalen labialen Vokale sind verschwunden: ${ }^{*} \bar{u}>\bar{u}$, ${ }^{*} k \breve{u}>k_{o} u,{ }^{*}$ pü $k>p u k,{ }^{*} \breve{u}(>* \breve{a})>a$;

2. die velaren weiten Vokale sind labialisiert worden: $* a \breve{a}>0$ (aber *kă- > $\left.\chi a-,{ }^{*} \breve{a} \chi>a \chi\right)$;

3. die palatalen weiten Vokale haben sich velarisiert: $* \bar{a}>a$, * $\ddot{a}>a$;

4. die velaren illabialen Engvokale sind verschwunden: ${ }^{*} \bar{\tau}>\bar{a}$, $*_{i}>i$;

(5. die langen Engvokale sind etwas offener geworden, ohne dass dies aber deren Status beeinflusste; das $o$ statt $\breve{\partial}$ ist wieder nur eine Frage der Lautbezeichnung).

Das so entstandene nordwogulische (So.) System lautet:

\begin{tabular}{ll}
$\bar{e}$ & $\bar{u}$ \\
$\bar{a}$ & $\bar{\jmath}$ \\
\hline$i$ & $u$ \\
$a$ & $o$
\end{tabular}

1.3. Wenn wir nun die Entwicklungstendenzen bis zum Nordostjakischen bzw. Nordwogulischen betrachten, kann wie folgt - festgestellt werden: 
I. 1. Das Nordostjakische ist unmittelbar aus einem (späturwestostjakischen) System mit drei Öffnungsgraden abzuleiten, - das Nordwogulische stammt dagegen aus einem (urwogulischen) System mit zwei Öffnungsgraden.

2. Die vollen (langen) Engvokale des Nordostjakischen waren mittelweit im frühreren System und verwandelten sich nur infolge der Reduzierung (Kürzung) der originellen vollen Engvokale in enge Glieder im System - im Nordwogulischen haben die Engvokale vom Urwogulischen an ausser $*_{\underline{\imath}}$ ihren Status bewahrt.

II. Die Norddialekte beider Sprachen haben auch gemeinsame Lautentwicklungstendenzen:

1. Die weiten Labialvokale sind zum Teil oder völlig durch Labialisierung entstanden: ostj. ${ }^{*} a>\jmath,{ }^{*} \breve{a} \times{ }^{*} \breve{o}>\check{o}$, wog. ${ }^{*} \bar{a}>$ $\bar{\jmath}, * \breve{a} \times *_{\breve{\jmath}}>o$.

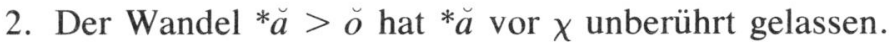

3. Die weiten illabialen Glieder der Systeme haben sich durch Velarisierung herausgebildet: ostj. $* \ddot{a}>a, * \breve{a}>\breve{a}$, wog. $* \bar{a}>\bar{a}\left(*^{-}>\bar{a}\right.$ auch!), * ${ }_{a}>a$.

Wir haben also dieselben grundlegenden Wandlungen, d.h. die Labialisierung und die Velarisierung der weiten Vokale in beiden Sprachen nachweisen können. Nun müsste noch die Frage beantwortet werden, ob sich diese Wandlungen voneinander abhängig, sich gegenseitig verstärkend vollzogen haben.

Steinitz hat die Labialisierung von urostj. ${ }^{*} a$ als einen Prozess betrachtet, der sich vom Süden nach Osten und Norden verbreitete (Steinitz 1950 a. 57) und der dann aller Wahrscheinlichkeit nach auch den Wandel $* \breve{a}>\breve{o}$ auslöste. $* \ddot{a}$ und $* \breve{a}(<* \breve{e})$ sind in fast allen ostjakischen Mundarten mehr oder weniger velarisiert worden, sie können aber nur in den näher behandelten Nordmundarten als phonematisch velare Vokale angesehen werden.

${ }^{*} \bar{a}$ hat sich fast im gesamten wogulischen Sprachgebiet labialisiert (vgl. Steinitz 1955. $170 \mathrm{ff}$.), deshalb könnte der im Norden eingetretene Lautwandel $* \bar{a}>\bar{j}$ kaum durch ostjakischen Einfluss - oder umgekehrt - erklärt werden. Die Velarisierung * $\bar{a}>\bar{a}, * \ddot{a}>a$ scheint im Vergleich zu den übrigen Dialekten eine nordwogulische Neuerung zu sein, da 
die Labialisierung von $* \bar{a}$ zu $* \bar{\jmath}$ im Westen der Velarisierung voranging.

Alles in allem: allein $* \bar{a}>\bar{a}, * \breve{a}>a$ und vielleicht $* \breve{a} \chi>a \chi$ sind die lautgeschichtlichen Ereignisse im Nordwogulischen, die wohl von den Lautentwicklungen in den übrigen Mundarten zu trennen und mit den ähnlichen Wandeln im Nordostjakischen zu verbinden sind. Die Velarisierung $* \bar{a}>\bar{a}, * \ddot{a}>a$ lässt sich aber auch durch innernordwogulische Entwicklung erklären, denn die "Leerstellen" mussten nach der Labialisierung $* \bar{a}>\bar{j}$, $*_{a}>o$ ausgefüllt werden.

Ich bin der Ansicht, dass die Labialisierung der velaren weiten Vokale im Wogulischen und im Ostjakischen - auch wenn sie in beiden Sprachen gleichzeitig, im 15. Jahrhundert, zu wirken beginnen sollte (STEINITZ 1955. 174) - kaum gemeinsamen Ursprungs sein kann, weil die Mehrheit der Wogulen zu dieser Zeit noch auf der europäischen Seite des Ural-Gebirges wohnte, also weit entfernt vom Entstehungsort der ostjakischen Labialisierung am Irtysch. Meiner Meinung nach ist die wogulische Labialisierung von der ostjakischen auch deshalb $\mathrm{zu}$ trennen, da sie alle weiten Langvokale im Ost- und Westwogulischen einbezogen hat; die Labialisierung von * $\overline{\ddot{a}}$ im Norden ist durch die Velarisierung verhindert worden. Der letztgenannte Prozess ist aber auch in einem Teil der östlichen und westlichen Mundarten zur Geltung gekommen. Für das Ostjakische ist dagegen die Entlabialisierung der palatalen Labialvokale charakteristisch und $* \ddot{a}$ hat in keiner einzigen Mundart eine labialisierte Fortsetzung. Übrigens ist die Velarisation in beiden Sprachen jungen Datums, so dass denkbar wäre, dass sich die nordwogulischen und nordostjakischen Velarisierungsprozesse gegenseitig hätten verstärken können.

2. Da nur einige Lautwandel nach der Auflösung der obugrischen Grundsprache stattfanden, weichen die Systeme der Konsonantenphoneme im Ostjakischen und im Wogulischen nicht wesentlich voneinander ab. Ein Teil der Veränderungen hatte gemeinsamen Ursprung in der Vergangenheit (z.B. ${ }^{*} k y>$ $\left.\chi \varnothing,{ }^{*} k \ddot{\gamma}>k \ddot{\gamma}\right)$, ein anderer Teil von ihnen hat - volkommen unabhängig voneinander - zu gleichen Ergebnissen geführt (z.B. urobugr. ${ }^{*} \check{c}>$ urostj. ${ }^{*} \check{c}[>$ nordostj. $\check{s},>\mathrm{O} s$ ], urobugr. 
$*_{\check{c}}>$ urwog. $*_{\check{s}}$ [ $>$ nordwog. s.]); diese haben ohne Zweifel verursacht, dass es trotz der auffallenden Unterschiede auch mehrere Übereinstimmungen gibt.

Die Konsonantenphoneme in den Norddialekten der obugrischen Sprachen sehen folgendermassen aus:

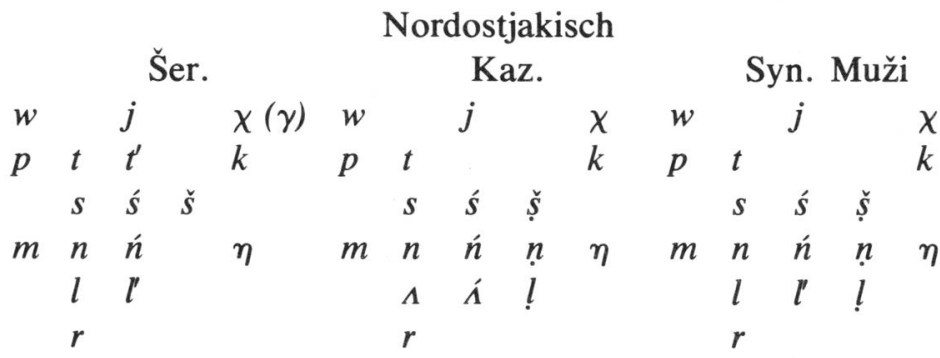

(Steinitz 1950 b. (vgl. Rédei 1968. (vgl. Steinitz $42-43)$ 11; KatZ 1975. 71) 1939/1975. 19 ff.; RÉDEI 1961. 8 ff.; KATZ 1975. 62)

Nordwogulisch

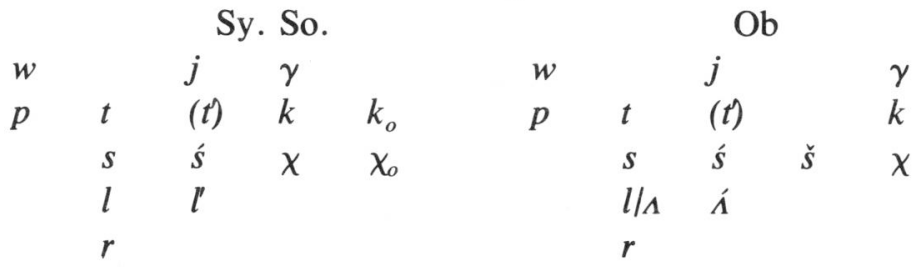

(vgl. Kálmán 1976. 22. ff.) (vgl. Kálmán 1976. 22 ff.)

1. In den nordostjakischen Mundarten ist das urostj. ${ }^{*} \check{c} \mathrm{zu} \breve{s}$ $(\mathrm{O} s)$ geworden, das urwog. $*_{s}$ ist wiederum im Nordwogulischen durch $s$ vertreten, nur in der Mundart am Ob kommt $\breve{s}$ sporadisch vor (KÁLMÁN 1976. 22, 24). Sein Neuentstehen und etymologisch nicht ganz konsequentes Auftreten ist höchstwahrscheinlich den benachbarten ostjakischen Mundarten zuzuschreiben.

2. In derselben wogulischen Mundart gibt es $A$ (neben dem l?) Und Á (Kálmán 1976. 22, 23); auch in diesem Falle kann man mit ostjakischem Einfluss rechnen. - Nach Kálmán tritt das $A$ 
vorwiegend vor $t$ auf (KÁLMÁN 1976. 23). Er hat sich jedoch nicht darüber geäussert, ob es sich um einen dem ostjakischen spirantischen $A$ ähnlichen Konsonanten handelt oder ob hier nur ein (halb)stimmloser Konsonant vorliegt. Eine solche Liquida kommt nämlich in anderen wogulischen Mundarten vor, z.B.

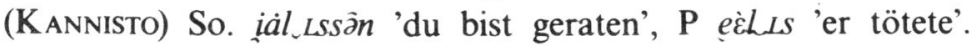

Im Bereich der Konsonanten gibt es $\mathrm{m}$. W. keine anderen Züge, die dem gegenseitigen Einfluss zuzuschreiben wären.

3. Ich möchte hervorheben, wenn sich die übereinstimmenden Vokalsysteme im Nordwogulischen und im Nordostjakischen auch grösstenteils aus inneren Lautentwicklungstendenzen erklären lassen, so gibt es jedoch in beiden Sprachen gemeinsame Züge, die kaum voneinander zu trennen sind:

1. Wog. So. $\bar{e}$ realisiert sich als $\bar{e}, \bar{\varepsilon}$, ebenso hat ostj. Kaz. $e$ die Varianten $e, \varepsilon$ (s. Steinitz 1937. 263-265, 1950 a. 15-16, 1955. 35, 220; RÉdEI 1968. 9-10; vgl. aber KATZ 1975. 67-69).

2. Urwog. $* \bar{a}$ ist - wie erwähnt - in So. zu $\bar{\jmath}$ geworden, dazu vgl. urostj. ${ }^{*} a>$ nordostj. o, im Ost- und Westwogulischen und in der LO-Mundart, die sich nicht unmittelbar an der wogulisch-ostjakischen Sprachgrenze befindet, ist die Fortsetzung $\operatorname{des}^{*} \bar{a}$ ein $\bar{o}$ (s. Steinitz 1955. 39).

3. Die Realisierung von nordwog. $u$ ist ein leicht palatalisierter Laut, ebenso wie die von nordostj. $\breve{u}$ (STEINITz 1950 a. 17, 1955. 35); das trifft auch für nordostj. $a$ und $a \mathbf{z u}$, während das nordwog. $a$ nur neben $k, \gamma$ und $j$ eine leicht palatalisierte Variante hat (STEINITZ 1937. 257, 1955. 32-33; KÁLMÁN 1976. 18).

Sowohl im Nordwogulischen als auch im Nordostjakischen kommt eine stimmhafte Variante des $s$ in intervokalischer und postkonsonantischer Stellung vor (KÁLMÁN 1976. 24; RÉdEI 1968. 12).

4. Aufgrund des obigen ist leicht einzusehen, dass die sekundären Übereinstimmungen, die auf gegenseitigen Einfluss zurückzuführen sind, ziemlich späten Ursprungs, sporadisch und gering sind. Die völlig übereinstimmenden Vokalphonemsysteme im Nordwogulischen und im Nordostjakischen sind zum Teil durch gleichgerichtete voneinander unabhängige Entwicklungstendenzen aus vollkommen verschiedenen Vorstufen ent- 
standen. Damit möchte ich selbstverständlich nicht behaupten, dass diese Systeme nicht als eine Erscheinungsform des Sprachbundes betrachtet werden können, sondern sich ausschliesslich durch innernordwogulische bzw. innerwest- und innernordostjakische Lautwandlungen ableiten lassen. Es ist darüber hinaus klar, dass es verfehlt ist, aus den gemeinsamen Zügen des Nordwogulischen und des Nordostjakischen den Schluss zu ziehen, sie seien nur die Folge der geographischen Nachbarschaft; aufgrund einer solcher falschen Schlussfolgerung ist es genauso falsch, die ehemalige wogulisch-ostjakische Sprachgemeinschaft, d.h. die obugrische Grundsprache als Zwischenstufe auf dem Wege vom Urugrischen zum Urwogulischen bzw. zum Urostjakischen zu bezweifeln, geschweige denn zu verneinen (natürlich gibt es nicht einmal in der Morphologie solche gemeinsamen Züge, die sich nicht mindestens auf das Urobugrische zurückführen lassen).

LÁszló Honti

\section{LITERATUR}

Gulya 1977: JÁNOS GulYA, Megjegyzések az ugor őshaza és az ugor nyelvek szétválása kérdéséről. In: ANTAL BARThA - KÁROly CZEglédy ANDRÁS RónA-TAS (Hrsg.), Magyar őstörténeti tanulmányok. Budapest. $115-121$.

Hont1 1978: LÁSzló Honti, Északi osztják szövegek szójegyzékkel. Nyelvtudományi Közlemények 80: 140-154.

Honti 1979: László Honti, Versuch einer Beschreibung des Phonembestandes im wogulischen Dialekt an der Pelymka. Vortrag beim 2. Symposion "Phonologie der uralischen Sprachen", 1-4. Oktober 1979, Mátrafüred.

Honti i.D.: LÁszló HontI, Geschichte des obugrischen Vokalismus der ersten Silbe. Budapest, i. D.

Kálmán 1976: Béla Kálmán, Wogulische Texte mit einem Glossar. Budapest.

Katz 1975: Hartmut Katz, Generative Phonologie und phonologische Sprachbünde des Ostjakischen und Samojedischen. München.

RÉdEI 1961: KÁROLY RADANOvics (RÉdEI), Északi-osztják nyelvtan. Nyelvtudományi Értekezések 31. Budapest.

RÉdEI 1968: KÁROLY Rédel. Nord-ostjakische Texte (Kazym-Dialekt) mit Skizze der Grammatik. Göttingen.

Steinitz 1937: Wolfgang Steinitz, Der Vokalismus des Sosva-Wogulischen. Sitzungsberichte der Gelehrten Estnischen Gesellschaft 1: 244-277. 
Steinitz 1939/1975: Wolfgang Steinitz, Ostjakische Volksdichtung und Erzählungen aus zwei Dialekten. 1. Teil. (Verhandlungen der Gelehrten Estnischen Gesellschaft 31.) Tartu 1939. (Ostjakologische Arbeiten. Band I.) Budapest-Berlin-Den Haag 1975.

Steinitz 1941/1976: Wolfgang Steinitz, Ostjakische Volksdichtung und Erzählungen aus zwei Dialekten. 2. Teil. Stockholm 1941. (Ostjakologische Arbeiten. Band II.) Budapest-Berlin-Den Haag 1976.

Steinitz 1950 a: Wolfgang Steinitz. Geschichte des ostjakischen Vokalismus. Berlin.

Steinitz 1950 b: WolfGang Steinitz, Ostjakische Grammatik und Chrestomathie mit Wörterverzeichnis. Leipzig.

Steinitz 1955: Wolfgang Steinitz, Geschichte des wogulischen Vokalismus. Berlin. 